\title{
LESSONS FOR COVID 19 ERA: IMPACT OF DELAYS IN SURGERY ON BIOCHEMICAL RECURRENCE-FREE SURVIVAL AND ADVERSE ONCOLOGICAL OUTCOMES IN PROSTATE CANCER PATIENTS
}

\author{
Bahadir Sahin ${ }^{1}$, Ozan Bozkurt ${ }^{2}$, Sinan Sözen ${ }^{3}$, Haluk Ozen $^{4}$, Bulent Akdogan ${ }^{5}$, Guven \\ Aslan $^{6}$, Volkan Izol ${ }^{7}$, Sumer Baltaci ${ }^{8}$, Levent Turkeri ${ }^{9}$, Serdar Çelik ${ }^{10}$, and Ilker Tinay ${ }^{11}$ \\ ${ }^{1}$ Marmara Universitesi Tip Fakultesi \\ ${ }^{2}$ Dokuz Eylül University \\ ${ }^{3}$ Gazi University \\ ${ }^{4}$ Hacettepe University Faculty of Medicine \\ ${ }^{5}$ Hacettepe University \\ ${ }^{6}$ Affiliation not available \\ ${ }^{7}$ Cukurova University Faculty of Medicine \\ ${ }^{8}$ Ankara University Faculty of Medicine \\ ${ }^{9}$ Acibadem University \\ ${ }^{10}$ Izmir Bozyaka Training and Research Hospital \\ ${ }^{11}$ Marmara University School of Medicine
}

April 14, 2021

\begin{abstract}
OBJECTIVE To assess the impact of the surgical delay for localized prostate cancer (PCa) on adverse pathological features and oncological outcomes. MATERIALS AND METHODS Patients who underwent surgery for localized prostate cancer were included from the Turkish Urooncology Association (TUA) Prostate Cancer database. A History of previous treatment or active surveillance (AS) were considered as exclusion criteria from the study. Patients were divided into two groups according the time period between the diagnosis and surgery; less than or equal to 90 days (group 1) or longer than 90 days (group 2). Surgical pathology results and oncological outcomes were compared between the two groups. RESULTS A total of 2454 out of 3646 patients were assessed. Pathological findings of the radical prostatectomy (RP) specimens were similar between two groups. However, there was slightly more seminal vesicle invasion in final surgical pathology in group $1(12.9 \%$ vs. $9.3 \%$, respectively $\mathrm{p}=0.042)$. 5-year biochemical recurrence free survival times were similar across all D'Amico risk categories between two groups. The regression analysis demonstrated the seminal vesicle invasion as the only factor affecting time to PSA progression in high-risk patients ( $\mathrm{p}<0.001 \mathrm{HR}: 2.51 \mathrm{CI}: 1,58-4,45)$. CONCLUSION In conclusion, our results in this large cohort suggest that surgical delay does not cause a deterioration for prostate cancer surgical outcomes even in high-risk group of patients. These findings may be helpful for planning the limited healthcare resources especially in conditions like the Covid-19 pandemic where the availability and optimal use of healthcare system resources is crucial.
\end{abstract}

LESSONS FOR COVID 19 ERA: IMPACT OF DELAYS IN SURGERY ON BIOCHEMICAL RECURRENCE-FREE SURVIVAL AND ADVERSE ONCOLOGICAL OUTCOMES IN PROSTATE CANCER PATIENTS 
Bahadır Şahin ${ }^{1}$, Ozan Bozkurt ${ }^{2}$, Sinan Sözen ${ }^{3}$, Haluk Özen ${ }^{4}$, Bülent Akdoğan ${ }^{4}$, Güven Aslan², Volkan İzol $^{5}$, Sümer Baltaci ${ }^{6}$, Levent Türkeri ${ }^{7}$, Serdar Çelik $^{8}$, İlker Tinay ${ }^{1}$

and Members of Turkish Urooncology Association

${ }^{1}$ Marmara University, School of Medicine, İstanbul, Turkey

2 Dokuz Eylül University, School of Medicine, İzmir, Turkey

3 Gazi University, School of Medicine, Ankara, Turkey

${ }^{4}$ Hacettepe University, School of Medicine, Ankara, Turkey

5 Çukurova University, School of Medicine, Adana, Turkey

6 Ankara University, School of Medicine, Ankara, Turkey

7 Acıbadem University, School of Medicine, İstanbul, Turkey

8 Bozyaka Research and Training Hospital, İzmir, Turkey

\section{ABSTRACT OBJECTIVE}

To assess the impact of the surgical delay for localized prostate cancer on adverse pathological features and oncological outcomes.

\section{MATERIALS AND METHODS}

Patients who underwent surgery for localized prostate cancer were included from the Turkish Urooncology Association (TUA) Prostate Cancer database. A History of previous treatment (radiotherapy or androgen deprivation therapy) or active surveillance (AS) were considered as exclusion criteria from the study. The date of prostate biopsy was regarded as the date of diagnosis and time to treatment was calculated as the number of days between the date of surgery and the diagnosis date. Patients were divided into two groups according the time period between the diagnosis and surgery; less than or equal to 90 days (group 1) or longer than 90 days (group 2). Surgical pathology results and oncological outcomes were compared between the two groups.

\section{RESULTS}

A total of 2454 out of 3646 patients were assessed, where in $79.8 \%$ of patients "diagnosis to surgery time" was less than or equal to 90 days. Groups were distributed similarly with respect to PSA value on diagnosis, Gleason grade groups of biopsy pathology and D'amico risk-group classification. Pathological findings of the RP specimens were similar between two groups with respect to surgical margin status, lymph node positivity and extracapsular extension. However, there was slightly more seminal vesicle invasion in final surgical pathology in group 1 ( $12.9 \%$ vs. $9.3 \%$, respectively $\mathrm{p}=0.042)$. Considering the low-risk patients, Gleason score upgrading was observed in $37.94 \%$ of group 1 compared to $30.56 \%$ of group 2 ( $\mathrm{p}=0.046$ ). 5 -year biochemical recurrence free survival times were similar across all D'Amico risk categories between two groups. In high-risk patients the need for adjuvant treatment was higher in group 1 (40.8\% vs. $25 \%$ respectively, $\mathrm{p}=0.023)$, whereas there was no statistically significant difference between groups with respect to metastasis- and PSA recurrence rate. The regression analysis demonstrated the seminal vesicle invasion as the only factor affecting time to PSA progression in high-risk patients ( $\mathrm{p}<0.001$ HR:2.51 CI: 1,58-4,45).

\section{CONCLUSION}

In conclusion, our results in this large cohort suggest that surgical delay does not cause a deterioration for prostate cancer surgical outcomes even in high-risk group of patients. These findings may be helpful for planning the limited healthcare resources especially in conditions like the Covid-19 pandemic where the availability and optimal use of healthcare system resources is crucial. 
Keywords: Prostate Cancer, Radical Prostatectomy, Surgical Delay, Urooncology, Survival INTRODUCTION

After a new diagnosis of localized $\mathrm{PCa}$, treatment options may range from AS to radical surgery in most cases. ${ }^{1}$ Patients are often encouraged to take a second opinion before they decide for the final treatment but this decision-making process could prolong the duration between the diagnosis and potential treatment. The current evidence on the impact of this waiting gap on the surgical and oncological outcomes of the localized $\mathrm{PCa}$ is conflicting. ${ }^{2,} 3$

The Covid-19 pandemic clearly delayed the surgical procedures due to overwhelming case load of infected patients in healthcare systems. Due to rapidly changing healthcare circumstances European Urological Association (EAU) and some national associations including Turkish Urooncology Association published recommendations during the pandemic and suggested a delay for definitive surgical treatment of PCa, between 3 to 6 months with respect to the risk groups of patients. ${ }^{4}$ Based on these recommendations, we aimed to assess the possible impact of the time duration between diagnosis and radical prostatectomy (RP) on the surgical and oncological outcomes of the disease.

\section{MATERIALS AND METHODS}

Data of patients who underwent RP as the initial treatment of PCa were reviewed retrospectively in this study. The data source was nationwide PCa database of Turkish Urooncological Association. A total of 3646 patients were found to be treated with RP for localized disease in the database and excluding patients with missing data, study population was downsized to 2454 patients. Patients were divided into two groups according to their waiting period between diagnosis and RP. The waiting period in respective groups was; Group 1: less than or equal to 3 months, Group 2: more than 3 months.

Based on D'amico classification system patients were stratified into low, intermediate and high-risk groups. The date of prostate biopsy was regarded as the diagnosis date and time to treatment is calculated as the number of days between the date of RP and the diagnosis date. Patients who received treatment for PCa (radiotherapy or androgen deprivation therapy etc.) prior to RP or patients who were first enrolled on AS protocol were excluded from the study.

All patients were diagnosed with either standard transrectal ultrasound guided biopsy or magnetic resonance (MR) guided fusion biopsy. All RPs were included in the study irrespective of the surgical approach (robot assisted, laparoscopic or open). Patients were operated by a senior urology staff in each participating center. Both biopsy and RP specimens were evaluated by a dedicated uro-pathologist in each center.

Biochemical recurrence which was defined as a prostate specific antigen (PSA) value measured greater than $0.2 \mathrm{ng} / \mathrm{ml}$ during the follow up after RP, is designated as the primary endpoint for this study. Secondary endpoints of the study were surgical parameters, pathological upgrading, metastasis on follow up and the need for additional treatments. For time-based analysis and comparison of oncological outcomes (biochemical recurrence free survival, need for adjuvant treatment or metastasis free survival), only patients with a follow up duration of more than 1 month was included in the statistical tests.

The study data were collected by REDCap data collection software developed by Vanderbilt University and licensed to Turkish Urooncology Association. ${ }^{5,6}$ All data are kept in a secure server and all personal information of patients were anonymized.

For statistical analysis Python Programming Language (Open source v3.7) was used with the help of pandas, matplotlib, numpy, scipy and lifelines ${ }^{7}$ libraries. JupyterLab (Open source v1.2.6) was used as the coding interface. The scalar variables were analyzed using visual (histograms, probability plots) and analytical methods (Kolmogorov-Simirnov/Shapiro-Wilk's tests) to determine whether or not they were normally distributed. Descriptive analyses were given as means and standard deviations when the variable was normally distributed. Medians and interquartile ranges were used if the variable was not normally distributed. For the 
comparison of scalar variables between two groups, t-test or Mann-Whitney U test were used for normally and non-normally distributed variables respectively.

Categorical variables were compared with Chi-square test between groups. If the assumptions of Chi-square do not hold due to low expected cell counts Fisher's exact test were used for the comparison of categorical variables. For biochemical recurrence free survival variable, Kaplan-Meier survival estimates were calculated. A separate log rank test was used to estimate independent effect of waiting duration group on time to biochemical recurrence. The possible factors identified in univariate analysis further evaluated with Cox regression. The proportional hazard assumption was assessed by means of residual analysis. For all statistical tests, $\mathrm{p}$ value below 0.05 was considered statistically significant.

\section{RESULTS}

Mean age of patients was $62.35 \pm 6.64$ years. There were 1959 and 495 patients in groups 1 and 2, respectively. Groups were distributed similarly with respect to PSA value on diagnosis, Gleason grade groups of biopsy pathology and D'amico risk group. (Table 1) Pre-diagnostic properties were similar between two groups for each D'amico risk group. (Table 2) Median elapsed time until treatment was 51 (38-65) days for group 1 and 119 (104-141) days for group 2.

Table 1: General patient characteristics:

\begin{tabular}{|c|c|c|c|c|}
\hline & & $\begin{array}{l}\text { Group } 1(<=3 \\
\text { Months) }\end{array}$ & $\begin{array}{l}\text { Group } 2(>3 \\
\text { Months) }\end{array}$ & $\mathrm{p}$ value \\
\hline Age & $($ Mean(SD)) & $62.26(6.63)$ & $62.52(6.77)$ & $0.176^{1}$ \\
\hline BMI & (Mean(SD)) & $27.14(3.77)$ & $27.02(2.97)$ & $0.7138^{1}$ \\
\hline PSA & (Median(IQR)) & $7.20(5.12-11)$ & $7.22(5.08-11.26)$ & $0.730^{2}$ \\
\hline \multirow{5}{*}{$\begin{array}{l}\text { Gleason Grade } \\
\text { Group n }(\%)\end{array}$} & 1 & $1017(51.91)$ & $284(57.37)$ & $0.133^{3}$ \\
\hline & 2 & $555(28.33)$ & $119(24.04)$ & \\
\hline & 3 & $191(9.75)$ & $52(10.51)$ & \\
\hline & 4 & $110(5.62)$ & $20(4.04)$ & \\
\hline & 5 & $86(4.39)$ & $20(4.04)$ & \\
\hline \multirow{3}{*}{$\begin{array}{l}\text { D'amico Group n } \\
(\%)\end{array}$} & Low Risk & $775(39.56)$ & $218(44.04)$ & $0.193^{3}$ \\
\hline & $\begin{array}{l}\text { Intermediate } \\
\text { Risk }\end{array}$ & $869(44.36)$ & $203(41.01)$ & \\
\hline & High Risk & $315(16.08)$ & $74(14.95)$ & \\
\hline \multirow{2}{*}{$\begin{array}{l}\text { Biopsy Type } \\
\text { n(\%) }\end{array}$} & Classical & $1823(93.06)$ & $471(95.15)$ & $0.092^{3}$ \\
\hline & MR Fusion & $136(6.64)$ & $124(4.85)$ & \\
\hline
\end{tabular}

BMI: Body Mass Index, SD: Standard Deviation, IQR: Interquartile Range, MR: Magnetic Resonance. ${ }^{1}$ Independent Samples t-test, ${ }^{2}$ Mann-Whitney U Test, ${ }^{3} \mathrm{x}^{2}$ Test

Table 2: General patient characteristics for each risk group

\begin{tabular}{lllllllll}
\hline & Low & Low & Low & \multicolumn{2}{c}{ Intermediatntermediatntermediałigh } & \multicolumn{2}{l}{ High } \\
& Risk & Risk & Risk & Risk & Risk & Risk & Risk & Risk \\
\hline & G1 & G2 & p & G1 & G2 & p & G1 & G2 \\
Age & 60.9 & 61.59 & $0,175^{1}$ & 62.94 & 63.25 & $0,545^{1}$ & 63.74 & 64.53 \\
Mean & $(6.53)$ & $(6.72)$ & & $(6.5)$ & $(6.82)$ & & $(6.61)$ & $(6.25)$ \\
(SD) & & & & & & & &
\end{tabular}




\begin{tabular}{|c|c|c|c|c|c|c|c|c|c|}
\hline & & \multirow{2}{*}{$\begin{array}{l}\text { Low } \\
\text { Risk }\end{array}$} & \multirow{2}{*}{$\begin{array}{l}\text { Low } \\
\text { Risk }\end{array}$} & \multirow{2}{*}{$\begin{array}{l}\text { Low } \\
\text { Risk }\end{array}$} & \multicolumn{4}{|c|}{ IntermediałntermediałntermediaÆŁigh } & \multirow{2}{*}{$\begin{array}{l}\text { High } \\
\text { Risk }\end{array}$} \\
\hline & & & & & Risk & Risk & Risk & Risk & \\
\hline \multicolumn{2}{|l|}{$\begin{array}{l}\text { BMI } \\
\text { Mean } \\
(\mathrm{SD})\end{array}$} & $\begin{array}{l}26.67 \\
(3.86)\end{array}$ & $\begin{array}{l}26.57 \\
(3.02)\end{array}$ & $0,850^{1}$ & $\begin{array}{l}27.24 \\
(3.8)\end{array}$ & $\begin{array}{l}27.32 \\
(3.18)\end{array}$ & $0,894^{1}$ & $\begin{array}{l}27.67 \\
(3.45)\end{array}$ & $\begin{array}{l}27.48 \\
(2.01)\end{array}$ \\
\hline \multicolumn{2}{|l|}{$\begin{array}{l}\text { PSA } \\
\text { Median } \\
\text { (IQR) }\end{array}$} & $\begin{array}{l}5.71(4.5 \\
-7.2)\end{array}$ & $\begin{array}{l}5.56(4.3 \\
-7.3)\end{array}$ & $0,317^{2}$ & $\begin{array}{l}8.7(5.8- \\
12.0)\end{array}$ & $\begin{array}{l}10.13 \\
(5.6- \\
12.5)\end{array}$ & $0,187^{2}$ & $\begin{array}{l}18.0(8.0 \\
-28.9)\end{array}$ & $\begin{array}{l}20.94 \\
(8.0- \\
27.0)\end{array}$ \\
\hline \multirow{5}{*}{$\begin{array}{l}\text { Gleason } \\
\text { Grade } \\
\text { Group n } \\
(\%)\end{array}$} & 1 & $\begin{array}{l}775 \\
(100.0)\end{array}$ & $\begin{array}{l}218 \\
(100.0)\end{array}$ & - & $\begin{array}{l}199 \\
(22.9)\end{array}$ & $\begin{array}{l}53 \\
(26.11)\end{array}$ & $0.302^{3}$ & $\begin{array}{l}43 \\
(13.65)\end{array}$ & $\begin{array}{l}13 \\
(17.57)\end{array}$ \\
\hline & 2 & - & - & & $\begin{array}{l}514 \\
(59.15)\end{array}$ & $\begin{array}{l}108 \\
(53.2)\end{array}$ & & $\begin{array}{l}41 \\
(13.02)\end{array}$ & $\begin{array}{l}11 \\
(14.86)\end{array}$ \\
\hline & 3 & - & - & & $\begin{array}{l}156 \\
(17.95)\end{array}$ & $\begin{array}{l}42 \\
(20.69)\end{array}$ & & $\begin{array}{l}35 \\
(11.11)\end{array}$ & $\begin{array}{l}10 \\
(13.51)\end{array}$ \\
\hline & 4 & - & - & & - & - & & $\begin{array}{l}110 \\
(34.92)\end{array}$ & $\begin{array}{l}20 \\
(27.03)\end{array}$ \\
\hline & 5 & - & - & & - & - & & $86(27.3)$ & $\begin{array}{l}20 \\
(27.03)\end{array}$ \\
\hline \multirow{2}{*}{$\begin{array}{l}\text { Biopsy } \\
\text { Type n } \\
(\%)\end{array}$} & St & $\begin{array}{l}729 \\
(94.06)\end{array}$ & $\begin{array}{l}209 \\
(95.87)\end{array}$ & $0.303^{3}$ & $\begin{array}{l}795 \\
(91.48)\end{array}$ & $\begin{array}{l}191 \\
(94.09)\end{array}$ & $0,219^{3}$ & $\begin{array}{l}299 \\
(94.92)\end{array}$ & $\begin{array}{l}71 \\
(95.95)\end{array}$ \\
\hline & MR & $46(5.94)$ & $9(4.13)$ & & $74(8.52)$ & $12(5.91)$ & & $16(5.08)$ & $3(4.05)$ \\
\hline
\end{tabular}

SD: Standard Deviation, BMI: Body Mass Index, IQR: Interquartile Range, St: Standard, MR: MR Guided G1: Group 1 ( $<=3$ Months), G2 = Group 2 ( $>3$ Months). ${ }^{1}$ Independent Samples t-test, ${ }^{2}$ Mann-Whitney $\mathrm{U}$ Test, ${ }^{3} \mathrm{x}^{2}$ Test

Surgical and pathological parameters including lymph node (LN) dissection, per-operative complications, type of RP, surgical margin (SM) status, LN positivity, extracapsular extension (ECE), seminal vesicle (SV) invasion and Gleason Grade Group at RP were in low, intermediate and high-risk patients ( $>>0.05)$ (Table 3 ). On the other hand, in intermediate risk patients, nerve sparing rate was found to be higher in group $1(\mathrm{p}=0.032)$. Additionally, in low-risk patients, in group 1, it was observed that Gleason Grade group significantly increased in RP pathology compared to biopsy pathology ( $\mathrm{p}=0.046$ ) (Table 3).

Table 3: Surgical and pathological Characteristics with respect to D'amico risk categories

\begin{tabular}{|c|c|c|c|c|c|c|c|c|c|}
\hline & & \multirow{2}{*}{$\begin{array}{l}\text { Low } \\
\text { Risk }\end{array}$} & \multirow{2}{*}{$\begin{array}{l}\text { Low } \\
\text { Risk }\end{array}$} & \multirow{2}{*}{$\begin{array}{l}\text { Low } \\
\text { Risk }\end{array}$} & \multicolumn{4}{|c|}{ Intermediałntermediałntermediałkigh } & \multirow{2}{*}{$\begin{array}{l}\text { High } \\
\text { Risk }\end{array}$} \\
\hline & & & & & Risk & Risk & Risk & Risk & \\
\hline & & G1 & G2 & $\mathrm{p}^{*}$ & G1 & G2 & $\mathrm{p}^{*}$ & G1 & G2 \\
\hline \multirow{2}{*}{$\begin{array}{l}\text { Nerve } \\
\text { Sparing } \\
\text { n (\%) }\end{array}$} & - & $\begin{array}{l}344 \\
(50.74)\end{array}$ & $\begin{array}{l}101 \\
(54.89)\end{array}$ & 0.317 & $\begin{array}{l}369 \\
(49.2)\end{array}$ & $92(58.6)$ & 0.032 & $\begin{array}{l}159 \\
(63.6)\end{array}$ & $\begin{array}{l}40 \\
(72.73)\end{array}$ \\
\hline & + & $\begin{array}{l}334 \\
(49.26)\end{array}$ & $\begin{array}{l}83 \\
(45.11)\end{array}$ & & $\begin{array}{l}381 \\
(50.8)\end{array}$ & $65(41.4)$ & & $91(36.4)$ & $\begin{array}{l}15 \\
(27.27)\end{array}$ \\
\hline \multirow{2}{*}{$\begin{array}{l}\text { LN Dis- } \\
\text { section } \\
\text { n (\%) }\end{array}$} & - & $\begin{array}{l}604 \\
(79.16)\end{array}$ & $\begin{array}{l}168 \\
(79.25)\end{array}$ & 0.979 & $\begin{array}{l}450 \\
(52.69)\end{array}$ & $\begin{array}{l}104 \\
(53.06)\end{array}$ & 0.926 & $\begin{array}{l}59 \\
(18.85)\end{array}$ & $\begin{array}{l}17 \\
(23.29)\end{array}$ \\
\hline & + & $\begin{array}{l}159 \\
(20.84)\end{array}$ & $\begin{array}{l}44 \\
(20.75)\end{array}$ & & $\begin{array}{l}404 \\
(47.31)\end{array}$ & $\begin{array}{l}92 \\
(46.94)\end{array}$ & & $\begin{array}{l}254 \\
(81.15)\end{array}$ & $\begin{array}{l}56 \\
(76.71)\end{array}$ \\
\hline
\end{tabular}




\begin{tabular}{|c|c|c|c|c|c|c|c|c|c|}
\hline & & \multirow{2}{*}{$\begin{array}{l}\text { Low } \\
\text { Risk }\end{array}$} & \multirow{2}{*}{$\begin{array}{l}\text { Low } \\
\text { Risk }\end{array}$} & \multirow{2}{*}{$\begin{array}{l}\text { Low } \\
\text { Risk }\end{array}$} & \multicolumn{4}{|c|}{ IntermediaIntermediaIntermediałEigh } & \multirow{2}{*}{$\begin{array}{l}\text { High } \\
\text { Risk }\end{array}$} \\
\hline & & & & & Risk & Risk & Risk & Risk & \\
\hline \multirow{3}{*}{$\begin{array}{l}\text { Per-op } \\
\text { Compli- } \\
\text { cation } \mathbf{n} \\
(\%)\end{array}$} & - & 717 & 186 & 0.513 & 796 & 187 & 0.572 & 297 & 67 \\
\hline & & $(95.09)$ & $(93.94)$ & & $(93.87)$ & $(94.92)$ & & $(95.81)$ & $(95.71)$ \\
\hline & + & $37(4.91)$ & $12(6.06)$ & & $52(6.13)$ & $10(5.08)$ & & $13(4.19)$ & $3(4.29)$ \\
\hline \multirow{3}{*}{$\begin{array}{l}\text { RP } \\
\text { Type } \mathbf{n} \\
(\%)\end{array}$} & $\mathbf{O}$ & 503 & 133 & 0.306 & 592 & 123 & 0.051 & 211 & \\
\hline & & $(65.92)$ & $(62.15)$ & & $(69.0)$ & $(61.81)$ & & $(68.73)$ & $(62.16)$ \\
\hline & $\mathbf{R} / \mathbf{L}$ & $\begin{array}{l}260 \\
(34.08)\end{array}$ & $\begin{array}{l}81 \\
(37.85)\end{array}$ & & $\begin{array}{l}266 \\
(31.0)\end{array}$ & $\begin{array}{l}76 \\
(38.19)\end{array}$ & & $\begin{array}{l}96 \\
(31.27)\end{array}$ & $\begin{array}{l}28 \\
(37.84)\end{array}$ \\
\hline \multirow{3}{*}{$\begin{array}{l}\text { Surgical } \\
\text { Margin } \\
\text { n (\%) }\end{array}$} & - & 571 & 159 & 0.494 & 554 & 126 & 0.709 & 122 & 28 \\
\hline & & $(76.03)$ & $(78.33)$ & & $(65.95)$ & $(67.38)$ & & $(40.13)$ & $(43.08)$ \\
\hline & + & $\begin{array}{l}180 \\
(23.97)\end{array}$ & $\begin{array}{l}44 \\
(21.67)\end{array}$ & & $\begin{array}{l}286 \\
(34.05)\end{array}$ & $\begin{array}{l}61 \\
(32.62)\end{array}$ & & $\begin{array}{l}182 \\
(59.87)\end{array}$ & $\begin{array}{l}37 \\
(56.92)\end{array}$ \\
\hline \multirow{2}{*}{$\begin{array}{l}\text { LN pos- } \\
\text { itivity } n \\
(\%)\end{array}$} & - & $\begin{array}{l}125 \\
(96.9)\end{array}$ & $\begin{array}{l}31 \\
(96.88)\end{array}$ & 0.994 & $\begin{array}{l}339 \\
(91.87)\end{array}$ & $\begin{array}{l}73 \\
(91.25)\end{array}$ & 0.855 & $\begin{array}{l}169 \\
(68.98)\end{array}$ & $\begin{array}{l}34 \\
(69.39)\end{array}$ \\
\hline & + & $4(3.1)$ & $1(3.12)$ & & $30(8.13)$ & $7(8.75)$ & & $\begin{array}{l}76 \\
(31.02)\end{array}$ & $\begin{array}{l}15 \\
(30.61)\end{array}$ \\
\hline \multirow[t]{2}{*}{$\begin{array}{l}\text { ECE n } \\
(\%)\end{array}$} & - & $\begin{array}{l}589 \\
(83.43)\end{array}$ & $\begin{array}{l}149 \\
(81.42)\end{array}$ & 0.519 & $\begin{array}{l}462 \\
(59.38)\end{array}$ & $\begin{array}{l}105 \\
(61.4)\end{array}$ & 0.626 & $\begin{array}{l}111 \\
(38.95)\end{array}$ & $\begin{array}{l}22 \\
(31.88)\end{array}$ \\
\hline & + & $\begin{array}{l}117 \\
(16.57)\end{array}$ & $\begin{array}{l}34 \\
(18.58)\end{array}$ & & $\begin{array}{l}316 \\
(40.62)\end{array}$ & $\begin{array}{l}66 \\
(38.6)\end{array}$ & & $\begin{array}{l}174 \\
(61.05)\end{array}$ & $\begin{array}{l}47 \\
(68.12)\end{array}$ \\
\hline \multirow{2}{*}{$\begin{array}{l}\text { SV } \\
\text { Invasion } \\
\text { n (\%) }\end{array}$} & - & $\begin{array}{l}726 \\
(96.41)\end{array}$ & $\begin{array}{l}197 \\
(98.5)\end{array}$ & 0.133 & $\begin{array}{l}729 \\
(87.52)\end{array}$ & $\begin{array}{l}167 \\
(88.83)\end{array}$ & 0.619 & $\begin{array}{l}192 \\
(62.95)\end{array}$ & $\begin{array}{l}49 \\
(72.06)\end{array}$ \\
\hline & + & $27(3.59)$ & $3(1.5)$ & & $\begin{array}{l}104 \\
(12.48)\end{array}$ & $\begin{array}{l}21( \\
11.17)\end{array}$ & & $\begin{array}{l}113 \\
(37.05)\end{array}$ & $\begin{array}{l}19 \\
(27.94)\end{array}$ \\
\hline \multirow{5}{*}{$\begin{array}{l}\text { Gleason } \\
\text { Grade } \\
\text { Group } \\
(\mathrm{RP}) \mathbf{n} \\
(\%)\end{array}$} & 1 & $\begin{array}{l}471 \\
(62.06)\end{array}$ & $\begin{array}{l}150 \\
(69.44)\end{array}$ & 0.162 & $\begin{array}{l}158 \\
(18.48)\end{array}$ & $44(22.0)$ & 0.123 & $17(5.54)$ & $5(7.04)$ \\
\hline & 2 & $\begin{array}{l}226 \\
(29.78)\end{array}$ & $\begin{array}{l}53 \\
(24.54)\end{array}$ & & $\begin{array}{l}479 \\
(56.02)\end{array}$ & $\begin{array}{l}102 \\
(51.0)\end{array}$ & & $\begin{array}{l}72 \\
(23.45)\end{array}$ & $\begin{array}{l}15 \\
(21.13)\end{array}$ \\
\hline & 3 & $\begin{array}{l}37 \\
(4.87)\end{array}$ & $\begin{array}{l}11 \\
(5.09)\end{array}$ & & $\begin{array}{l}155 \\
(18.13)\end{array}$ & $\begin{array}{l}41 \\
(20.5)\end{array}$ & & $\begin{array}{l}77 \\
(25.08)\end{array}$ & $\begin{array}{l}10 \\
(14.08)\end{array}$ \\
\hline & 4 & $16(2.11)$ & $1(0.46)$ & & $45(5.26)$ & $5(2.5)$ & & $\begin{array}{l}49 \\
(15.96)\end{array}$ & $\begin{array}{l}21 \\
(29.58)\end{array}$ \\
\hline & 5 & $9(1.19)$ & $1(0.46)$ & & $18(2.11)$ & $8(4.0)$ & & $\begin{array}{l}92 \\
(29.97)\end{array}$ & $\begin{array}{l}20 \\
(28.17)\end{array}$ \\
\hline $\begin{array}{l}\text { Gleason } \\
\text { Grade }\end{array}$ & - & $\begin{array}{l}471 \\
(62.06)\end{array}$ & $\begin{array}{l}150 \\
(69.44)\end{array}$ & 0.046 & $\begin{array}{l}643 \\
(75.2)\end{array}$ & $\begin{array}{l}156 \\
(78.0)\end{array}$ & 0.406 & $\begin{array}{l}236 \\
(76.87)\end{array}$ & $\begin{array}{l}52 \\
(73.24)\end{array}$ \\
\hline
\end{tabular}




\begin{tabular}{lllllllll}
\hline & Low & Low & Low & \multicolumn{2}{c}{ Intermediafatermediafatermediałigh } & \multicolumn{2}{l}{ High } \\
& Risk & Risk & Risk & Risk & Risk & Risk & Risk & Risk \\
\hline+ & 288 & 66 & & 212 & $44(22.0)$ & 71 & 19 \\
& $(37.94)$ & $(30.56)$ & & $(24.8)$ & & & $(23.13)$ & $(26.76)$ \\
\hline
\end{tabular}

LN: Lymph Node, RP: Radical Prostatectomy, O: Open, R/L: Robot Assisted/Laparoscopic, ECE: Extracapsular Extension, SV: Seminal Vesicle, G1: Group 1 ( $<=3$ Months), G2 = Group 2 ( $>3$ Months). ${ }^{*} \mathrm{x}^{2}$ Test

When we compared 2 groups according to surgical and pathological findings, we found no significant differences between 2 groups regarding all parameters, except SV invasion, nerve sparing rate and surgical modality in final pathology. Significantly more SV invasion in final RP pathology was found in group 1. ( $12.9 \%$ vs. $9.3 \%$, respectively $\mathrm{p}=0.042)$ Also more nerve sparing ( $48.0 \%$ vs 41.1 , respectively $\mathrm{p}=0,014)$ and open surgeries $(67.7 \%$ vs $62.0 \%, \mathrm{p}=0.014)$ were performed in group 1. (Table 4)

Table 4: Surgical and pathological characteristics of groups

\begin{tabular}{|c|c|c|c|c|}
\hline & & G1 & G2 & p value* \\
\hline \multirow{2}{*}{$\begin{array}{l}\text { Nerve Sparing } n \\
(\%)\end{array}$} & - & $872(51.97)$ & $233(58.84)$ & 0.014 \\
\hline & + & $806(48.03)$ & $163(41.16)$ & \\
\hline \multirow{2}{*}{$\begin{array}{l}\text { LN Dissection n } \\
(\%)\end{array}$} & - & $1113(57.67)$ & $289(60.08)$ & 0.337 \\
\hline & + & $817(42.33)$ & $192(39.92)$ & \\
\hline \multirow{3}{*}{$\begin{array}{l}\text { Per-op } \\
\text { Complication } \mathbf{n} \\
(\%)\end{array}$} & - & $1810(94.67)$ & $440(94.62)$ & 0.971 \\
\hline & & & & \\
\hline & + & $102(5.33)$ & $25(5.38)$ & \\
\hline \multirow[t]{2}{*}{ RP Type n (\%) } & $\mathrm{O}$ & $1306(67.74)$ & $302(62.01)$ & 0.017 \\
\hline & $\mathbf{R} / \mathbf{L}$ & $622(32.26)$ & $185(37.99)$ & \\
\hline \multirow{2}{*}{$\begin{array}{l}\text { Surgical Margin } \\
\text { n (\%) }\end{array}$} & - & $1247(65.8)$ & $313(68.79)$ & 0,226 \\
\hline & + & $648(34.2)$ & $142(31.21)$ & \\
\hline \multirow{2}{*}{$\begin{array}{l}\text { LN positivity n } \\
(\%)\end{array}$} & - & $633(85.2)$ & $138(85.71)$ & 0.866 \\
\hline & + & $110(14.8)$ & $23(14.29)$ & \\
\hline \multirow[t]{2}{*}{$\operatorname{ECE} n(\%)$} & - & $1162(65.69)$ & $276(65.25)$ & 0.865 \\
\hline & + & $607(34.31)$ & $147(34.75)$ & \\
\hline \multirow{2}{*}{$\begin{array}{l}\text { SV Invasion n } \\
(\%)\end{array}$} & - & $1647(87.1)$ & $413(90.57)$ & 0.042 \\
\hline & + & $244(12.9)$ & $43(9.43)$ & \\
\hline \multirow{5}{*}{$\begin{array}{l}\text { Gleason Grade } \\
\text { Group (RP) n } \\
(\%)\end{array}$} & 1 & $646(33.63)$ & $199(40.86)$ & 0.053 \\
\hline & 2 & $777(40.45)$ & $170(34.91)$ & \\
\hline & 3 & $269(14.0)$ & $62(12.73)$ & \\
\hline & 4 & $110(5.73)$ & $27(5.54)$ & \\
\hline & 5 & $119(6.19)$ & $29(5.95)$ & \\
\hline $\begin{array}{l}\text { ISUP Upgrade } n \\
(\%)\end{array}$ & - & $1350(70.28)$ & $358(73.51)$ & 0.160 \\
\hline
\end{tabular}




\begin{tabular}{llll}
\hline & G1 & G2 & p value* \\
\hline+ & $571(29.72)$ & $129(26.49)$ & \\
\hline
\end{tabular}

LN: Lymph Node, RP: Radical Prostatectomy, O: Open, R/L: Robot Assisted/Laparoscopic, ECE: Extracapsular Extension, SV: Seminal Vesicle, G1: Group 1 ( $<=3$ Months), G2 = Group 2 ( $>3$ Months). ${ }^{*} \mathrm{x}^{2}$ Test

Oncological outcomes like the need for adjuvant treatment, PSA recurrence and development of metastasis on follow up were similar between groups in low and intermediate risk patients. (Table 5) In high risk patients adjuvant treatment need rate was higher in group $1(\mathrm{p}=0.023)$ whereas there was no statistically significant difference between groups with respect to metastasis rate and PSA recurrence rate. (Table 5) Estimated 5-year biochemical recurrence free survival rates were similar in both groups for all three risk categories. ( $\mathrm{p}=0.700,0.932$ and 0.085 respectively) (Figure 1)

Table 5: Oncological Outcomes

\begin{tabular}{|c|c|c|c|c|c|c|c|c|}
\hline & \multirow{2}{*}{$\begin{array}{l}\text { Low } \\
\text { Risk }\end{array}$} & \multirow{2}{*}{$\begin{array}{l}\text { Low } \\
\text { Risk }\end{array}$} & \multirow{2}{*}{$\begin{array}{l}\text { Low } \\
\text { Risk }\end{array}$} & \multicolumn{4}{|c|}{ Intermediałntermediafntermediałkigh } & \multirow{2}{*}{$\begin{array}{l}\text { High } \\
\text { Risk }\end{array}$} \\
\hline & & & & Risk & Risk & Risk & Risk & \\
\hline & G1 & G2 & $\mathrm{p}^{*}$ & G1 & G2 & $\mathrm{p}^{*}$ & G1 & G2 \\
\hline \multirow{2}{*}{$\begin{array}{l}\text { PSA } \\
\text { Recur- } \\
\text { rence } \mathbf{n} \\
(\%)\end{array}$} & $\begin{array}{l}589 \\
(89.92)\end{array}$ & $\begin{array}{l}148 \\
(91.36)\end{array}$ & 0.582 & $\begin{array}{l}629 \\
(85.69)\end{array}$ & $\begin{array}{l}129 \\
(86.0)\end{array}$ & 0.922 & $\begin{array}{l}178 \\
(67.17)\end{array}$ & $48(80.0)$ \\
\hline & $\begin{array}{l}66 \\
(10.08)\end{array}$ & $\begin{array}{l}14 \\
(8.64)\end{array}$ & & $\begin{array}{l}105 \\
(14.31)\end{array}$ & $\begin{array}{l}21 \\
(14.0)\end{array}$ & & $\begin{array}{l}87 \\
(32.83)\end{array}$ & $\begin{array}{l}12 \\
(20.0)\end{array}$ \\
\hline \multirow{2}{*}{$\begin{array}{l}\text { Additional- } \\
\text { Therapy } \\
\text { n (\%) }\end{array}$} & $\begin{array}{l}603 \\
(92.06)\end{array}$ & $\begin{array}{l}147 \\
(90.74)\end{array}$ & 0.583 & $\begin{array}{l}609 \\
(82.97)\end{array}$ & $\begin{array}{l}121 \\
(80.67)\end{array}$ & 0.498 & $\begin{array}{l}157 \\
(59.25)\end{array}$ & $45(75.0)$ \\
\hline & $\begin{array}{l}52 \\
(7.94)\end{array}$ & $\begin{array}{l}15 \\
(9.26)\end{array}$ & & $\begin{array}{l}125 \\
(17.03)\end{array}$ & $\begin{array}{l}29 \\
(19.33)\end{array}$ & & $\begin{array}{l}108 \\
(40.75)\end{array}$ & $\begin{array}{l}15 \\
(25.0)\end{array}$ \\
\hline \multirow{2}{*}{$\begin{array}{l}\text { Metastasis- } \\
\text { on } \\
\text { Follow } \\
\text { Up n } \\
(\%)\end{array}$} & $\begin{array}{l}649 \\
(99.08)\end{array}$ & $\begin{array}{l}160 \\
(98.77)\end{array}$ & 0.712 & $\begin{array}{l}713 \\
(97.14)\end{array}$ & $\begin{array}{l}144 \\
(96.0)\end{array}$ & 0.460 & $\begin{array}{l}243 \\
(91.7)\end{array}$ & $\begin{array}{l}55 \\
(91.67)\end{array}$ \\
\hline & $6(0.92)$ & $2(1.23)$ & & $21(2.86)$ & $6(4.0)$ & & $22(8.3)$ & $5(8.33)$ \\
\hline
\end{tabular}

G1: Group 1 (<=3 Months), G2 = Group 2 ( $>3$ Months $).{ }^{*} \mathrm{x}^{2}$ Test

Figure 1: Kaplan-Meier estimates of biochemical recurrence-free survival 

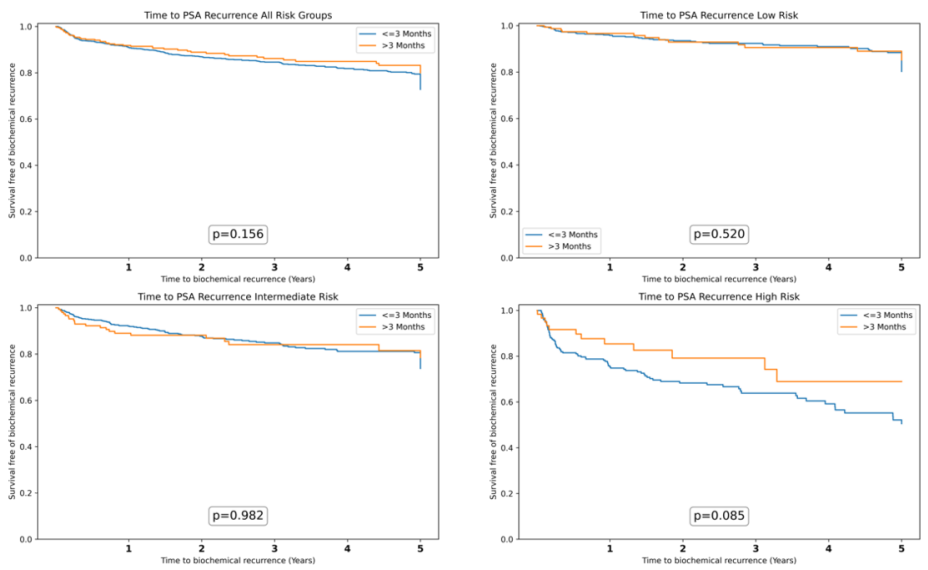

p values: Log Rank Test.

High risk patients were further analyzed for the factors affecting biochemical recurrence free survival with multivariate analysis. Cox regression analysis including patients' waiting period, PSA value at the time of diagnosis, Gleason grade groups in prostate biopsy and radical prostatectomy specimens, presence of positive surgical margins and/or having SV invasion demonstrated the main factor affecting time to PSA progression in high risk patients as SV invasion $(\mathrm{p}<0.001 \mathrm{HR}: 2.51 \mathrm{CI}: 1,58-4,45)$. Other factors including time to surgery $(\mathrm{p}=0.156 \mathrm{HR}: 0.63 \mathrm{CI}: 0.33-1.19)$ did not have any statistically significant impact on outcome.

\section{DISCUSSION}

In patients with localized PCa, our results showed that surgical margin status, LN positivity and the presence of ECE were similar irrespective of waiting period between the diagnosis and RP, however there was a slightly more SV invasion rate in final RP pathology of patients with a "diagnosis to surgery time" less than or equal to 90 days. Similarly in low-risk subgroup, Gleason Grade group upgrading in RP was found to be significantly higher in group 1 compared to group 2. However, 5-year biochemical recurrence free survival rates were similar in all three risk categories between the two study groups. In high-risk patients, the need for adjuvant treatment was higher in group 1 and the regression analysis demonstrated that the only factor affecting time to PSA progression in high-risk patient population was SV invasion at the RP pathology.

In the present study, median time elapsed until treatment was 119 (104-141) days in group 2 and biochemical recurrence rate in high risk patient category at this cut-off point (22.6\%) was not statistically significant. $(\mathrm{p}=0.605$, Data not shown) Since the number of patients with a delay time of more than 4 months were limited in our study, it was not possible to determine a safe cut-off time. On the other hand, our results clearly indicated a safe waiting period up to 4 months. In order to comment on longer delay times studies including more patients with longer wait times are needed.

This is one of the studies with the largest number of patients on this subject. Since our data source is a nationwide database with patient information from reference centers all around the country, results could be generalized to general population in Turkey. Most of the published data on surgical delay times are derived from AS studies and conducted in low/intermediate risk groups. ${ }^{8}, 9$ There are few studies which include high-risk PCa patients but there is no uniformity in these studies with respect to risk classification criteria or time cut-off levels for surgical delay. ${ }^{10,11}$ Our study is also one of the few studies that included all of the risk groups. Patients who first enrolled in AS are excluded from our study which enabled us to asses time delay more objectively, especially in low-risk patients. 
Decision making about a treatment modality from the available options could be challenging for PCa patients, especially in localized disease. Also, as the Covid-19 pandemic demonstrated, in some situations public health regulations and status of health care systems could necessitate a delay in the treatment of patients. In most cases guidelines specifies the treatment options but has no comment on the timing of the treatment. For most of the cancer types there are debates on the time intervals and their effect on the oncological outcomes. ${ }^{12}$

Urological cancers are no exception on these debates and there are some studies investigating the effect of treatment delay in all urological cancers. Urothelial cancer which is a typical example, has proven to be adversely effected by the delay in treatment. Hollenback et.al. showed that more than $25 \%$ of patients had delays of more than 3 months from the first occurrence of hematuria to definitive diagnosis. They also demonstrated that patients with a longer delay needed more radical interventions including cystectomy and the mortality rate was higher in this group. ${ }^{13}$ On the other hand Wallace et.al. showed that, although a shorter delay in the hospital did not have a profound impact, longer delays in treatment due to factors associated with referral patterns cause worse outcomes. ${ }^{14}$

Testicular cancer was traditionally regarded as a urological emergency. Although there are some reports demonstrating the adverse effect of treatment and diagnosis delay in testicular cancer ${ }^{15,16}$, there are also studies that do not show any benefit of early surgery in seminomatous tumors. ${ }^{17}$, 18 Since timing of surgery is still controversial, there are no recommendations regarding the time of orchiectomy in the guidelines of EAU. Physicians also encouraged to offer sperm cryopreservation to the patients before orchiectomy in EAU guidelines, which could result in short delays in surgery. ${ }^{19}$

The data on treatment delays in renal cell carcinoma is even more limited. There are reports indicating that the delays in surgery has no impact on disease specific survival for small $(<4 \mathrm{~cm})$ renal masses. ${ }^{20,}{ }^{21}$ On the other hand, for renal masses more than $4 \mathrm{~cm}$ diameter surgery is recommended before one month in a recent review, although there is no objective evidence demonstrating the adverse effect of late surgery. ${ }^{22}$

Studies on the effect of surgical delay on PCa prognosis are also conflicting. In 2017 a Canadian study demonstrated that even in patients with high-risk disease, surgical wait time does not affect pathological outcome after robot assisted RP (RARP). ${ }^{23}$ Furthermore, a recent study conducted on 2303 men demonstrated that in unfavorable prognosis group a waiting period up to 6 months does not have any adverse effect on disease outcomes. ${ }^{11}$ Similarly, Morini et. al. showed that even in patients who had waiting period of more than 6 months before treatment, oncological results were not adversely effected. ${ }^{24}$ There are other studies which reported similar results and could not find association between surgical delay time and disease progression. ${ }^{25-27}$

Despite the results of some studies showing no effect of surgical delay times in PCa patients, there are also contrasting reports which demonstrate delay in time to treatment as an unfavorable prognostic factor. In a series of 1111 low-risk PCa patients O'Brien et. al. reported worse oncological outcomes for patients who waited more than 6 months for the surgery. ${ }^{28}$ A more recent study performed on RARP patients showed that increased duration from biopsy to surgery may lead to more biochemical recurrence in high-risk group. ${ }^{10}$

Our study in concordance with the previous studies, showed no correlation between the surgical delay and biochemical recurrence free survival in overall patient cohort and after risk group stratification. Although some studies demonstrated a worse outcome with prolonged surgical delay time in high risk patients, those reports were limited in patient numbers and had different time cut-offs. Absence of a standardized definition on duration of cut-off in studies may be the underlying reason for contrasting results in different studies.

Limitations of the study

Our study is not without limitations. First, this is a retrospective analysis and selection bias could be an issue like all studies of this kind. Second, this is a multi-institutional study and there are more than one operating surgeons who performed the operations and uro-pathologists who assessed RP specimens. Both surgeon experience and surgical technique (open, robot assisted or laparoscopic) might have influenced patient outcomes. Our study marked the date of prostate biopsy as the reference point to calculate the time 
to surgery, but this may not always reflect the actual duration of the disease, since patients' first admission to the physician and timing of the prostate biopsy may differ between various institutions even within the same hospital system. An attempt to overcome bias, we stratified patients based on their D'Amico risks groups in order to provide more balanced distribution between cohorts. The median delay time in patient cohort waited longer than 90 days was nearly 4 months in our study. This is a limiting factor for this study in order to comment on longer delay times and specify a safe surgical time cut-off.

\section{CONCLUSION}

This study is one of the largest studies investigating the effect of surgical delay on the outcome of PCa using data originating from daily-practice. Our results indicate that patients could be reassured delays in time to surgery would not result in an adverse outcome even in high-risk group. Our findings may also be helpful in planning of limited healthcare resources especially in conditions like the Covid-19 pandemic.

\section{REFERENCES}

1. Mottet N, van den Bergh RCN, Briers E, et al. EAU - ESTRO - ESUR - SIOG Guidelines on Prostate Cancer 2020. European Association of Urology Guidelines. 2020 Edition. Vol presented at the EAU Annual Congress Amsterdam 2020. Arnhem, The Netherlands: European Association of Urology Guidelines Office; 2020 .

2. Katims AB, Razdan S, Eilender BM, et al. Urologic oncology practice during COVID-19 pandemic: A systematic review on what can be deferrable vs. nondeferrable. Urologic Oncology: Seminars and Original Investigations : Elsevier; 2020.

3. Boorjian SA, Bianco Jr FJ, Scardino PT, Eastham JA. Does the time from biopsy to surgery affect biochemical recurrence after radical prostatectomy? BJU international. 2005;96:773-776.

4. Çelik S, Tinay İ, Narter F, et al. Management of Patients with Urological Cancers in Turkey during the COVID-19 Pandemic: Recommendations of Uro-oncology Association. Bull Urooncol.2020;19:100-103.

5. Harris PA, Taylor R, Thielke R, Payne J, Gonzalez N, Conde JG. Research electronic data capture (REDCap) - a metadata-driven methodology and workflow process for providing translational research informatics support. Journal of biomedical informatics.2009;42:377-381.

6. RT PH, Minor B, Elliott V. REDCap Consortium, the REDCap Consortium: building an international community of software partners.J Biomed Inform. 2019.

7. Davidson-Pilon C, Kalderstam J, Jacobson N, et al. CamDavidsonPilon/lifelines: v0. 25.4. Zenodo: Genève, Switzerland. 2020.

8. Deka R, Courtney PT, Parsons JK, et al. Association Between African American Race and Clinical Outcomes in Men Treated for Low-Risk Prostate Cancer With Active Surveillance. JAMA.2020;324:17471754 .

9. Richard PO, Timilshina N, Komisarenko M, et al. The long-term outcomes of Gleason grade groups 2 and 3 prostate cancer managed by active surveillance: Results from a large population-based cohort. Canadian Urological Association Journal. 2020;14.

10. Zanaty M, Alnazari M, Ajib K, et al. Does surgical delay for radical prostatectomy affect biochemical recurrence? A retrospective analysis from a Canadian cohort. World journal of urology.2018;36:1-6.

11. Gupta N, Bivalacqua TJ, Han M, et al. Evaluating the impact of length of time from diagnosis to surgery in patients with unfavourable intermediate-risk to very-high-risk clinically localised prostate cancer. $B J U$ international. 2019;124:268-274.

12. Neal RD, Tharmanathan $P$, France $B$, et al. Is increased time to diagnosis and treatment in symptomatic cancer associated with poorer outcomes? Systematic review. British Journal of Cancer.2015;112:S92-S107. 
13. Hollenbeck BK, Dunn RL, Ye Z, et al. Delays in diagnosis and bladder cancer mortality. Cancer. 2010;116:5235-5242.

14. Wallace D, Bryan R, Dunn J, Begum G, Bathers S, Group WMUR. Delay and survival in bladder cancer. BJU international.2002;89:868-878.

15. Akdas A, Kirkah Z, Remzi D. The Role of Delay in Stage-Ill Testicular Tumors. European urology. 1986;12:151-153.

16. Huyghe E, Muller A, Mieusset R, et al. Impact of diagnostic delay in testis cancer: results of a large population-based study.European urology. 2007;52:1710-1716.

17. Dieckmann K-P, Becker T, Bauer H. Testicular tumors: presentation and role of diagnostic delay. Urologia internationalis. 1987;42:241-247.

18. Meffan P, Delahunt B, Nacey J. The value of early diagnosis in the treatment of patients with testicular cancer. The New Zealand Medical Journal. 1991;104:393-394.

19. Laguna MP, Albers P, Algaba F, et al. EAU Guidelines on Testicular Cancer 2020. European Association of Urology Guidelines. 2020 Edition. Vol presented at the EAU Annual Congress Amsterdam 2020. Arnhem, The Netherlands: European Association of Urology Guidelines Office; 2020.

20. Volpe A, Cadeddu JA, Cestari A, et al. Contemporary management of small renal masses. European urology.2011;60:501-515.

21. Van Poppel H, Joniau S. Is surveillance an option for the treatment of small renal masses? European urology.2007;52:1323-1330.

22. Bourgade V, Drouin SJ, Yates DR, et al. Impact of the length of time between diagnosis and surgical removal of urologic neoplasms on survival. World journal of urology. 2014;32:475-479.

23. Zanaty M, Alnazari M, Lawson K, et al. Does surgical delay for radical prostatectomy affect patient pathological outcome? A retrospective analysis from a Canadian cohort. Canadian Urological Association Journal. 2017;11:265.

24. Morini MA, Muller RL, de Castro Junior PCB, de Souza RJ, Faria EF. Time between diagnosis and surgical treatment on pathological and clinical outcomes in prostate cancer: does it matter? World journal of urology. 2018;36:1225-1231.

25. Korets R, Seager CM, Pitman MS, Hruby GW, Benson MC, McKiernan JM. Effect of delaying surgery on radical prostatectomy outcomes: a contemporary analysis. BJU international.2012;110:211-216.

26. Redaniel MT, Martin RM, Gillatt D, Wade J, Jeffreys M. Time from diagnosis to surgery and prostate cancer survival: a retrospective cohort study. BMC cancer. 2013;13:559.

27. Khan MA, Mangold LA, Epstein JI, Boitnott JK, Walsh PC, Partin AW. Impact of surgical delay on long-term cancer control for clinically localized prostate cancer. The Journal of urology.2004;172:1835-1839.

28. O'Brien D, Loeb S, Carvalhal GF, et al. Delay of surgery in men with low risk prostate cancer. The Journal of urology.2011;185:2143-2147. 